\title{
Total artificial heart: surgical technique in the patient with normal cardiac anatomy
}

\author{
Joshua S. Chung ${ }^{1}$, Dominic Emerson ${ }^{2}$, Dominick Megna ${ }^{2}$, Francisco A. Arabia ${ }^{3}$ \\ ${ }^{1}$ Department of Cardiothoracic Surgery, Loma Linda University Health, Loma Linda, California, USA; ${ }^{2}$ Department of Cardiac Surgery, Smidt \\ Heart Institute, Cedars-Sinai Medical Center, Los Angeles, California, USA; ${ }^{3}$ Advanced Heart Program, Banner University Medical Group, Phoenix, \\ Arizona, USA \\ Correspondence to: Joshua S. Chung, MD. Department of Cardiothoracic Surgery, Loma Linda University Health, 11175 Campus Street, Suite 21121, \\ Loma Linda, California, USA. Email: joshchung@gmail.com.
}

\begin{abstract}
Heart failure is a complex, growing problem with significant morbidity and mortality. Though heart transplantation remains the gold standard treatment for end-stage heart failure, there remains a national shortage of donor hearts. Mechanical circulatory support has provided an additional option for clinicians to support patients for the purposes of bridging patients to transplantation or to be used for destination therapy purposes. Despite generally favorable outcomes with univentricular support, in a subset of patients with biventricular heart failure, an isolated left ventricular assist device is not sufficient. Right ventricular failure has a negative impact on patient survival if not identified and treated promptly. The Total Artificial Heart (TAH) is the only Food and Drug Administration (FDA) approved artificial heart used for bridging patients to transplantation. Outcomes in patients who undergo implantation of the TAH at experienced centers have been good and reproducible.
\end{abstract}

Keywords: Total artificial heart (TAH); biventricular heart failure; mechanical circulatory support

Submitted Dec 04, 2019. Accepted for publication Jan 18, 2020.

doi: $10.21037 /$ acs.2020.02.09

View this article at: http://dx.doi.org/10.21037/acs.2020.02.09

\section{Introduction}

Heart failure is a complex, growing problem with an estimated prevalence of 6.2 million American adults affected between 2013 and 2016 (1). Though medical optimization has improved survival, heart transplantation remains the gold standard for definitive therapy of eligible patients with end-stage heart failure (2). In the United States, 2,841 adult heart transplantations were performed in 2017 , and although the number of transplants has steadily increased over the last decade, there is still a significant shortage of donor hearts with over 2,700 patients currently on the waiting list (3).

More recently, the advent of durable mechanical devices has provided an additional option for these patients as they can be implanted for the purpose of bridging to transplantation or being used for destination therapy (4-6). Utilization of these durable devices has steadily increased, with the proportion of candidates with ventricular assist devices at listing increasing to 50\% in 2016 (7). Despite generally favorable outcomes with univentricular support, in a subset of patients with biventricular heart failure, an isolated left ventricular assist device (LVAD) alone is insufficient (8-11). Additionally, the incidence of right ventricular failure after LVAD implantation is $9 \%$ to $30 \%$ $(12,13)$ and leads to considerable morbidity and mortality (14-16). Due to the challenges presented by biventricular dysfunction, there is a distinct need for durable biventricular support in select patients (17-19). The SynCardia Total Artificial Heart (TAH, SynCardia Systems, United States) was created to provide biventricular support and is the only FDA approved artificial heart for bridging patients to transplant in the United States (20).

\section{Description of the TAH}

The TAH is a pneumatically driven pulsatile system used for orthotropic replacement of the native ventricles and 


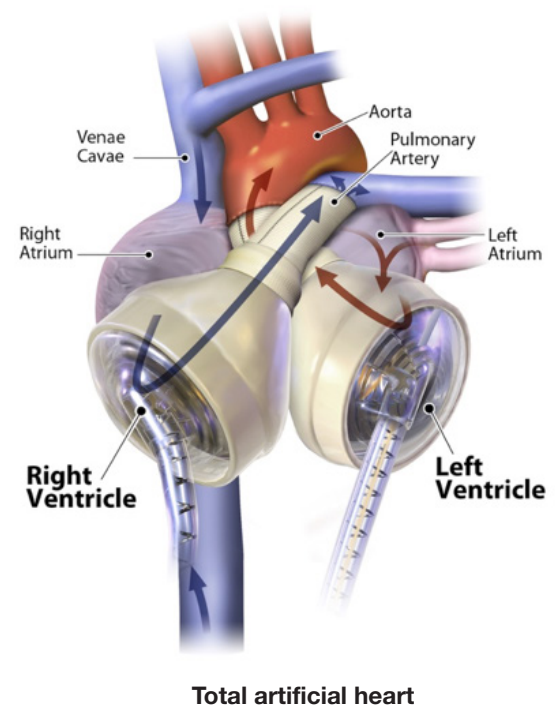

Figure 1 SynCardia total artificial heart.

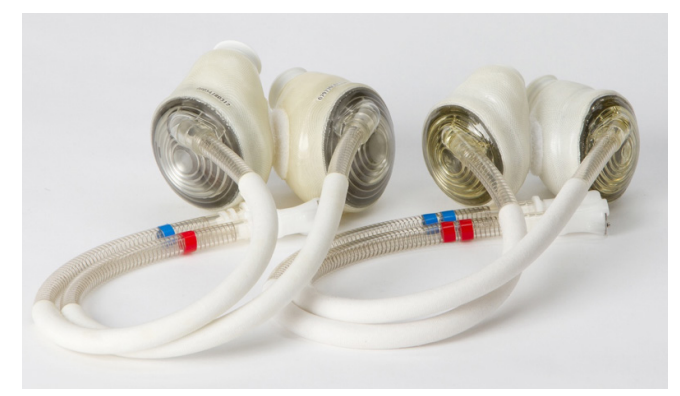

Figure 270 and $50 \mathrm{cc}$ total artificial heart.

four valves in case of end-stage biventricular heart failure (Figure 1). It consists of two independent artificial ventricles and is available in two sizes, 70 and $50 \mathrm{cc}$ (Figure 2). The $70 \mathrm{cc}$ has a maximum stroke volume of $70 \mathrm{~mL}$ and is capable of generating a cardiac output of up to $9.5 \mathrm{~L} / \mathrm{min}$. The $50 \mathrm{cc}$ has a maximum stroke volume of $50 \mathrm{~mL}$ and is capable of generating a flow rate of up to $7.5 \mathrm{~L} / \mathrm{min}$. Each ventricle is divided by a multi-layer flexible polyurethane diaphragm that separates the blood chamber from the air chamber. The polyurethane ventricles are connected by Velcro, which allows for flexible positioning inside the chest cavity. The direction of blood flow is controlled by four SynHall (formerly Medtronic Hall) tilting disc valves that are mounted in the inflow and outflow ports of each artificial ventricle. The cannula of each artificial ventricle acts as a pneumatic conduit from the driver to the ventricles. Each cannula is partially covered with velour fabric to promote

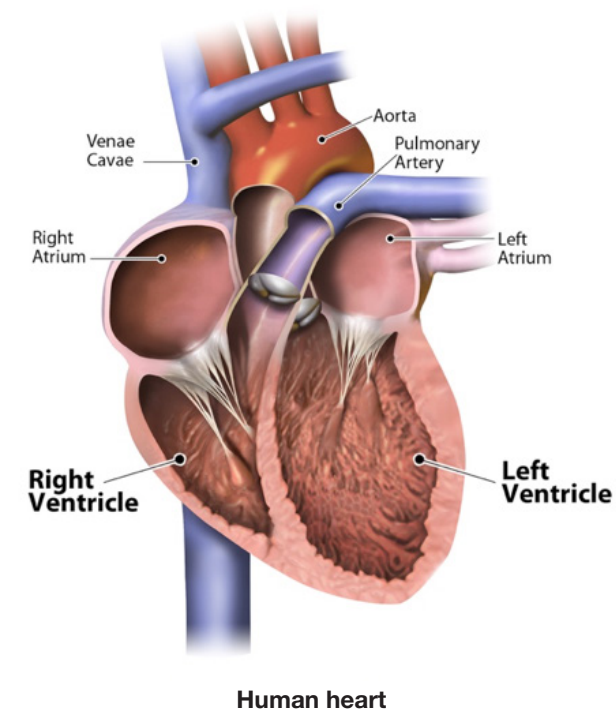

tissue ingrowth. They are tunneled through the chest wall and attached to drivelines that are connected to the external pneumatic driver. Initially, while the patient is recovering from implantation, he or she is connected to the $\mathrm{C} 2$ driver (Figure 3). After the patient is clinically stable, they can be switched to the Freedom Driver, a smaller, lighter pneumatic pump for the TAH, which allows increased mobility (Figure 4) and the ability to be discharged home.

\section{Indications/contraindications}

The TAH should be considered for patients with end-stage restrictive cardiomyopathy, irreversible biventricular failure, allograft failure, rejection or heart transplant vasculopathy, massive myocardial infarction, or refractory ventricular tachycardia. Anatomic considerations do exist since the $70 \mathrm{cc}$ ventricle requires at least a $10 \mathrm{~cm}$ distance from the T10 vertebral body to the border of the sternum. The $50 \mathrm{cc}$ requires a distance of at least $8 \mathrm{~cm}$. Contraindications include patients with small chest cavity size, chronic cardiac cachexia, chronic failure of end organs incompatible with recovery, high risk of intracranial hemorrhage secondary to cerebral arterial aneurysms or acute ischemic strokes, those who cannot be anticoagulated, and those who are ineligible for heart transplantation.

\section{Surgical technique}

The operation to implant a TAH in a patient with normal 


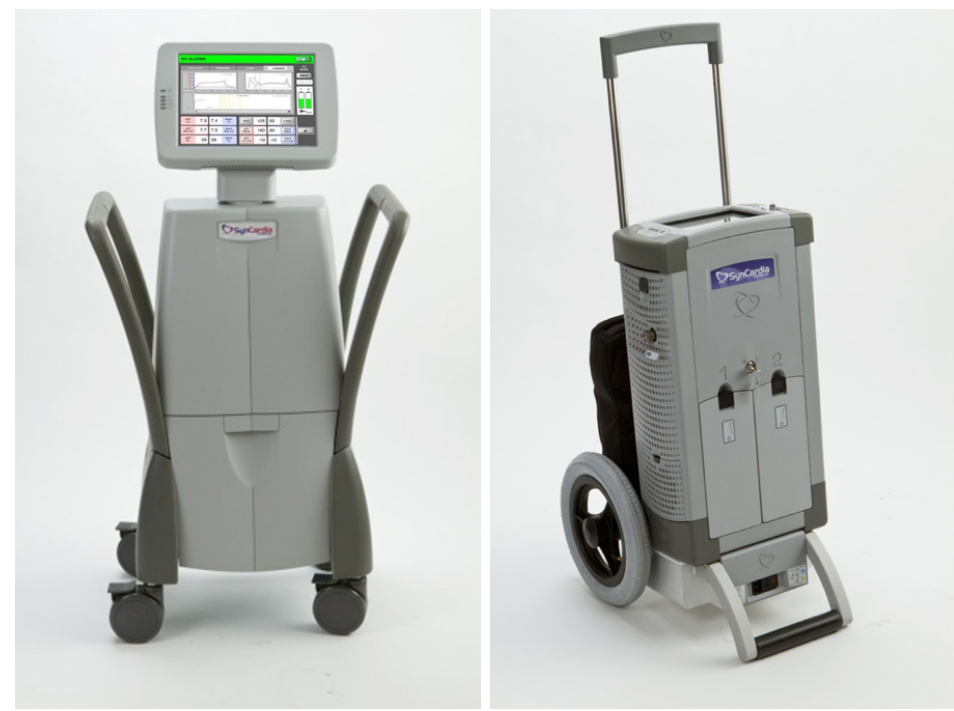

Figure 3 C2 external driver.

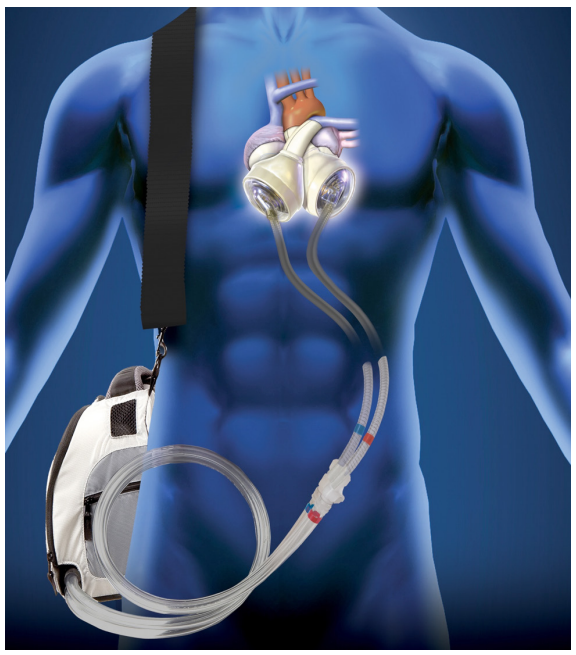

Figure 4 Freedom Driver allows increased mobility so patients can be discharged to home.

cardiac anatomy begins with a standard median sternotomy. The pericardium is opened, and visualization of cardiac function is done on the table and via transesophageal echocardiogram (TEE) to confirm the need for TAH implantation. Two small incisions approximately 2 inches below the coastal margin along the left mid clavicular line are made for the two driveline cannulas. The cannulas are then tunneled with the assistance of a cut $36 \mathrm{~F}$ chest tube. Care is taken to introduce the driveline at an angle towards the left pleural space to allow the ventricles to sit properly.

The aortic and pulmonary outflow grafts are then prepared by spraying a layer of CoSeal Surgical Sealant (Baxter International, United States) to these grafts to ensure hemostasis. The ventricles and the grafts are then soaked in rifampin and full heparinization is then given.

Mediastinal dissection is then minimally performed in order to maintain planes for dissection for the future transplant. The distal ascending aorta is cannulated and bicaval cannulation is performed via the right atrium in order to assist with leaving the superior vena cava (SVC) and inferior vena cava (IVC) intact. Tapes are placed around the SVC and IVC. $\mathrm{CO}_{2}$ is used to flood the surgical field. After adequate activated clotting time (ACT) is achieved, patient is initiated on cardiopulmonary bypass. The patient's temperature is allowed to drift to 34 degrees Celsius.

The aorta is then cross clamped and a cardiectomy is performed by initially incising the right ventricle (RV) along the right atrioventricular (AV) groove, and carrying this incision circumferentially around the base of the $\mathrm{RV}$, taking care to leave the tricuspid annulus intact. The residual ventricular muscle should be thin and approximately $1 \mathrm{~cm}$ wide. The tricuspid valve is excised leaving 1-2 $\mathrm{mm}$ of tricuspid leaflet remaining attached to the annulus. This is often used as an added buttress for the future anastomosis. The left ventricle (LV) is then similarly trimmed to just above the mitral annulus, again taking care to leave the mitral annulus intact. The mitral valve is also 


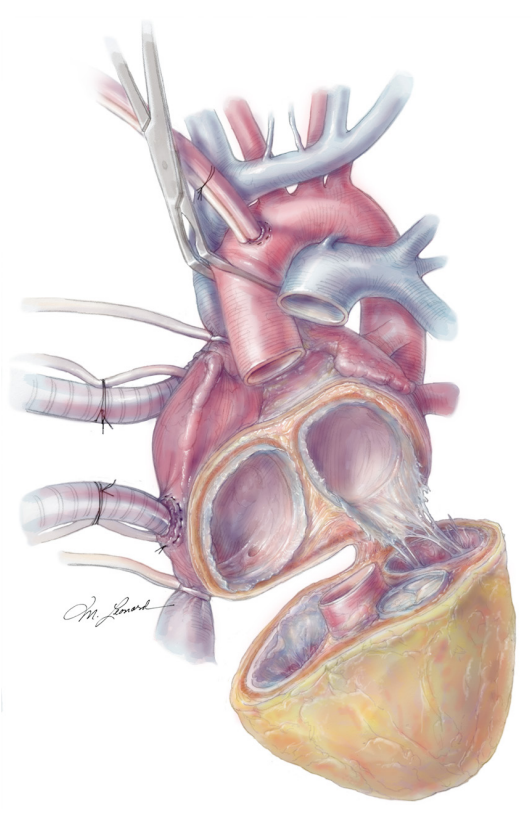

Figure 5 Cardiectomy for total artificial heart implantation.

resected to the same level. The aorta and pulmonary artery are then transected just above the aortic and pulmonic valves respectively, ensuring adequate remaining aorta and pulmonary artery for the transplant to follow (Figure 5). The coronary sinus is then identified and oversewn. The atrial septum should be carefully inspected for an atrial septal defect and closed if present.

The quick connects are then prepared by cutting the cuff circumferentially, leaving a small rim approximately $5 \mathrm{~mm}$ in width. Using 3-0 proline, the quick connects are then anastomosed to the respective AV valve annuli in a running fashion (Figure 6). A U-stitch at the 3'oclock and 9'oclock position allows the surgeon to stabilize the quick connect within the ventricle. Additionally, a Lone Star retractor may also facilitate implantation by stabilizing and elevating the heart. Care is taken while sewing in the quick connect to ensure that the suture passes through the cuff only and includes the annulus on the atrial side. Reinforcement stitches are then placed as needed. The left ventricle quick connect anastomosis is performed first, followed by the right ventricle anastomosis (Figures 6,7). At times when the patient's muscle is friable, a strip of felt pledget covered with a thin GOR-TEX (W.L. Gore and Associates, USA) membrane can be used to reinforce the anastomosis. The ventricles are then inserted but not connected to accurately measure the length of the aortic and pulmonary grafts. The pulmonary graft is longer than the aortic graft as it

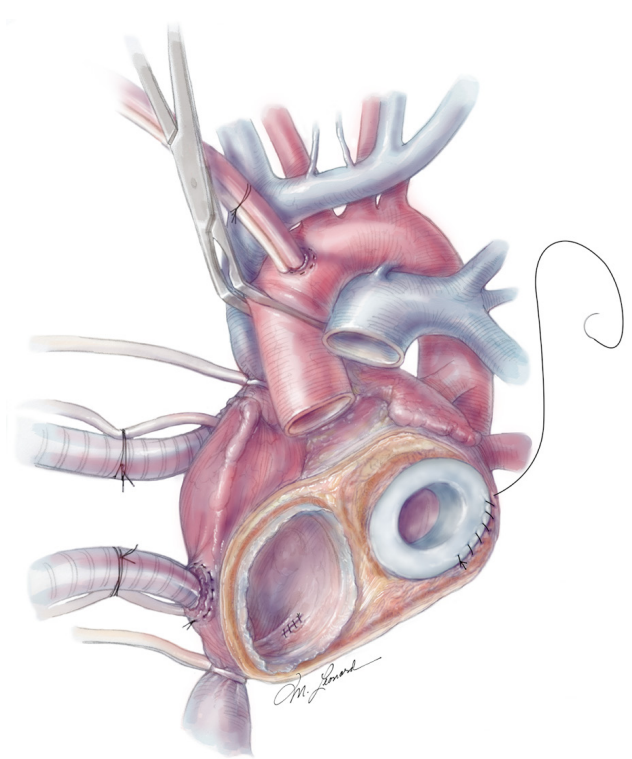

Figure 6 Left quick connect anastomosis.

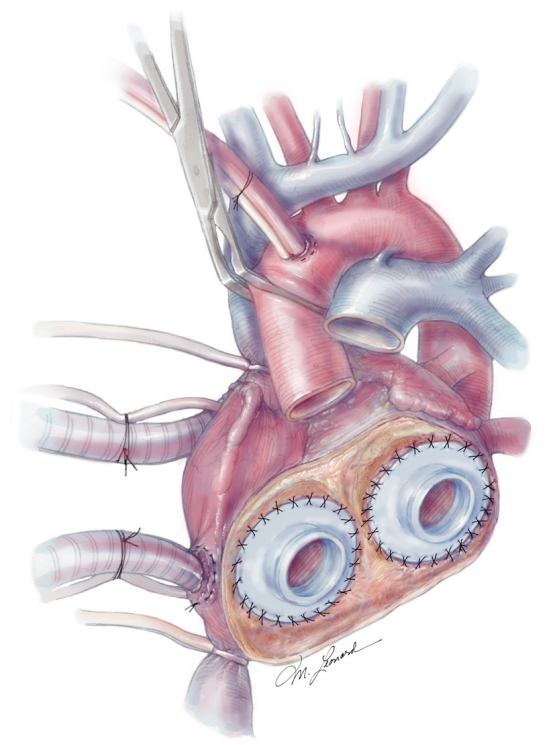

Figure 7 Left and right quick connect anastomoses.

overrides the aortic graft. Careful sizing is critical to avoid kinking or stretching both grafts.

The aortic and pulmonary graft anastomoses are then completed (Figure 8). The ventricles and outflow graft anastomoses are then leak tested with diluted methylene blue. When testing the left ventricle, manual compression of the pulmonary veins is performed with the surgeon's hand. Additionally, a vascular clamp is placed on the 


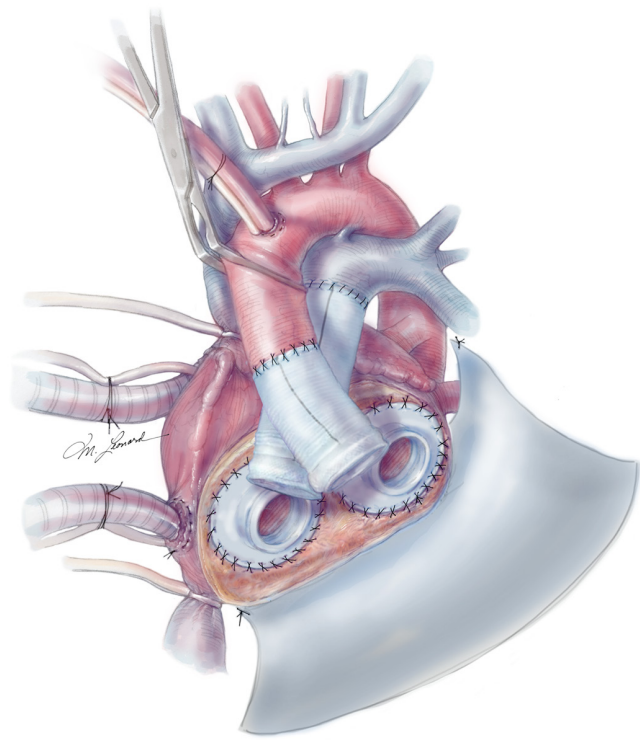

Figure 8 All anastomoses are completed prior to connecting the TAH. A Gortex membrane is inserted along the left pulmonary veins.

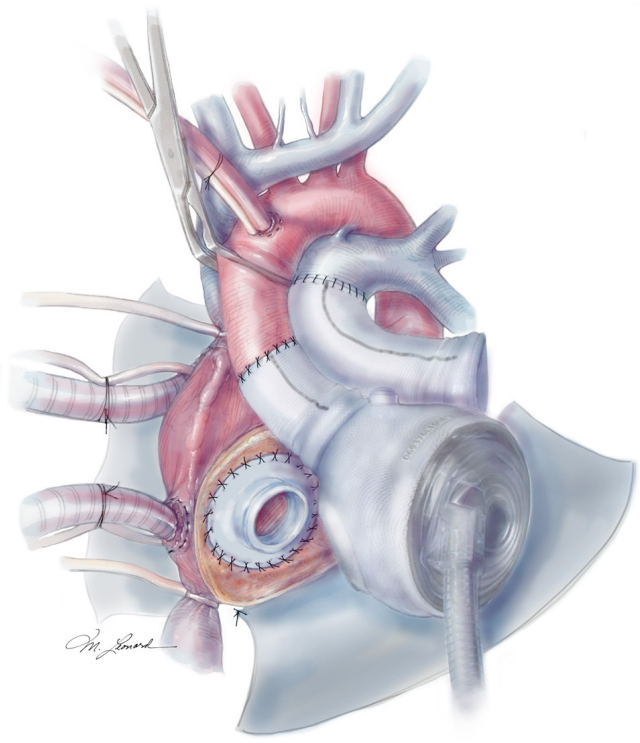

Figure 9 Insert left ventricle first.

pulmonary artery distal to the anastomosis to assess for leaks. Reinforcement sutures are then placed as necessary. A $0.1 \mathrm{~mm}$ GOR-TEX membrane is then placed to the level of the left pulmonary veins (Figure 8). Prior to the ventricles being placed within the pericardium, two heavy ties are placed around the knobs in the ventricles. The left ventricle

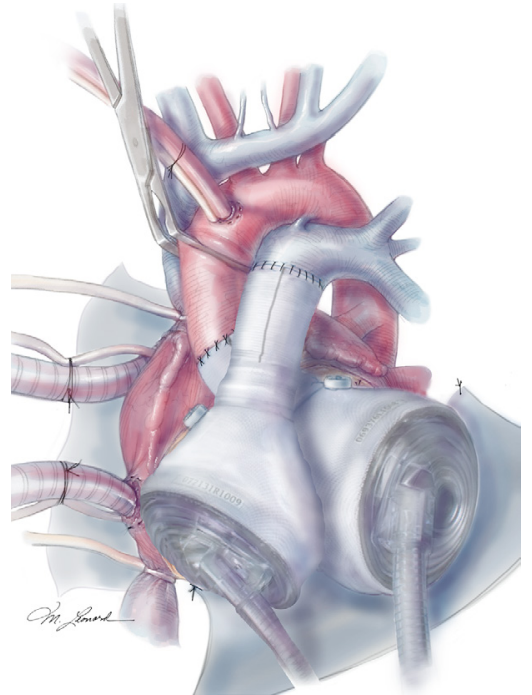

Figure 10 TAH fully implanted.

is then inserted first (Figure 9). Three blunt needle drivers are placed at 10 o'clock and 2 o'clock positions along the quick connect and the ventricle is inserted. The aortic quick connect is then attached in a similar manner. Prior to completing the aortic connection, the left ventricle is allowed to fill up with blood and irrigation is placed within the ventricle to evacuate as much air as possible. The right ventricle is then inserted and positioned (Figure 10). Snares are then released, and the right ventricle is filled with blood as the pulmonic connection is performed. The TAH is then connected to the $\mathrm{C} 2$ driver and the aortic cross clamp is removed. Aggressive de-airing from a root vent in the ascending aorta is essential during this period, and TEE is used to watch for air in the aorta. The TAH is then started with single manual beats to de-air. After adequate de-airing is performed, the TAH is set at a rate of 60 beats/minute. TAH support is then increased as the patient is weaned off bypass. Post bypass normal parameters are: rate 120-135, left pressure 180-200, right pressure 80-100, and vacuum 10-15.

Careful evaluation of the pulmonary veins should be performed, and velocities are inspected with TEE to ensure unobstructed return to the left atrium. The IVC is also inspected to rule out compression from the ventricles. In addition to evaluation after device start-up, these structures should be evaluated at closure as well. Due to the significantly decompensated state of these patients, coagulopathy is a concern and the chest is routinely left open for one day. At the time of final closure, several maneuvers are performed to ensure ease of re-entry at 
the time of transplant as follows: GOR-TEX membrane is placed along the right ventricle and atrium to the right pulmonary veins. Silastic "blue" bands are placed around the aortic anastomosis and pulmonary anastomosis, as well as the IVC and at times, the SVC. These bands help facilitate identification and isolation of these structures on reentry. The sternum is then closed with sternal wires with a thin silastic membrane between the wires and the sternum to facilitate the redo sternotomy.

If the anteroposterior diameter of the chest is small, the left pleural space can be opened and the device rotated down and laterally towards the left pleura. The surgeon should be cognizant of the left pleural veins in this approach, as they may be torted, impeding blood return. Often a large tacking suture can be placed to the chest wall to fix the ventricle in place. If the mediastinal space is large, saline implants or tissue expanders can be placed to preserve the mediastinal space, as contracture of the pericardium can often occur otherwise.

\section{Anticoagulation}

Anticoagulation in TAH is extremely critical as patients are now exposed to the risk of four mechanical valves and extensive foreign body contact of blood. Warfarin and aspirin are the mainstays of therapy in the long term, with heparin utilized in the early postoperative period. Adequate levels of anticoagulation should be determined by a combination of standard lab values (INR, PTT) and TEG. Institutional policies vary for exact parameters utilized, and further research as to the optimal levels is ongoing.

\section{Surgical outcomes}

Copeland et al. (21) first described their experience of a cohort of patients that received TAH with the intent to bridge to transplantation. In their study, 81 patients underwent implantation with $79 \%$ survival to transplantation and an overall one-year survival after TAH implantation of $70 \%$.

Recently, Arabia et al. (22) reported on the INTERMACS registry outcomes of TAH implantation from 2006-2017. Survival at 1 year was less at $53.9 \%$, and $33.9 \%$ at 2 years. Twenty-four percent of patients were able to be discharged out of the hospital, with $91 \%$ of those to home. Overall survival to transplantation was $53 \%$. The total mortality rate was $34 \%$ with the most common causes of death being multi-system organ failure, followed by neurologic injury and then by elective withdrawal of support. Risk factors for death included older age, renal failure, lower albumin, and implantation at a low-volume center. Patients who required pre-implant dialysis had a particularly high mortality rate, with greater than $50 \%$ 6-month mortality. Furthermore, experience in implanting and managing these patients was found to be critical. Comparing the centers that placed more than ten TAH implants to those that did less, the 12 -month survival was $64.8 \%$ vs. $36.7 \%$, respectively. The most common causes of early adverse events ( $<3$ months) were bleeding and infection. Late adverse events included infection and device malfunction.

\section{Future devices}

Although the current TAH is undergoing destination therapy studies, currently TAH therapy is not approved for patients who are not transplant candidates. However, new artificial heart platforms are currently undergoing investigation. With the advent of these new platforms, this goal will hopefully one day be realized.

Recently, CARMAT, a bioprosthetic artificial heart, was developed and underwent a pilot study in France (23). Several features make this artificial heart attractive as it is made of biocompatible materials with the potential to reduce thromboembolic risks, it provides pulsatility, and it is the first intelligent artificial heart to offer an immediate, automated response to the patient's physiologic needs. An early feasibility trial in the United States is pending as center enrollment is ongoing.

BiVACOR is an artificial heart created in Houston, Texas, and is currently undergoing animal studies (24). This artificial heart operates by two centrifugal impellers placed on a single magnetically levitated rotor that can provide high flows over $12 \mathrm{~L}$. The pump also has a smart controller that adapts to the changes in the patient's activities. This artificial heart is small enough that it can be implanted in a child.

Cleveland Clinic has also developed a continuous flow artificial heart that is valveless, sensorless and provides pulsatility (25). It is able to passively self-balance the left and right circulations without electronic intervention. It is currently undergoing animal studies.

\section{Conclusions}

Severe end-stage biventricular function is a complicated 
problem for clinicians. The TAH is the only FDA approved artificial heart available. Although these patients are often critically ill, there has been good success in bridging patients to transplantation with the current TAH. The future is bright for mechanical circulatory support as new artificial hearts are being created with the continuing advances in technology.

\section{Acknowledgments}

None.

\section{Footnote}

Conflicts of Interest: The authors have no conflicts of interest to declare.

\section{References}

1. Benjamin EJ, Munter P, Alonso A, et al. Heart Disease and Stroke Statistics -- 2019 Update: A Report From the American Heart Association. Circulation 2019;139:e56-e528.

2. Fuchs M, Schibilsky D, Zeh W, et al. Does the heart transplant have a future? Eur J Cardiothorac Surg 2019;55:i38-48.

3. Colvin M, Smith JM, Hadley N, et al. OPTN/SRTR 2017 Annual Data Report: Heart. Am J Transplant 2019;19 Suppl 2:323-403.

4. Rose EA, Gelihns AC, Moskowitz AJ, et al. Long-Term Use of a Left Ventricular Assist Device for End-Stage Heart Failure. N Engl J Med 2001;345:1435-43.

5. Rogers JG, Bulter J, Lansman SL, et al. Chronic mechanical circulatory support for inotrope-dependent heart failure patients who are not transplant candidates: results of the INTrEPID Trial. J Am Coll Cardiol 2007;50:741-7.

6. Slaughter MS, Rogers JG, Milano CA, et al. Advanced Heart Failure Treated with Continuous-Flow Left Ventricular Assist Device. N Engl J Med 2009;361:2241-51.

7. Khush KK, Cherikh WS, Chambers DC, et al. The International Thoracic Organ Transplant Registry of the International Society for Heart and Lung Transplantation: Thirty-fifth Adult Heart Transplantation Report - 2019; Focus Theme: Multiorgan Transplantation. J Heart Lung Transplant 2018;37:1155-68.

8. Ochiai Y, McCarthy PM, Smedira NG, et al. Predictors of severe right ventricular failure after implantable left ventricular assist device insertion: analysis of 245 patients. Circulation 2002;106:I-1989-202.

9. Alturi P, Goldstone AB, Fairma AS, et al. Predicting Right Ventricular Failure in the Modern Continuous Flow Left Ventricular Assist Device Era. Ann Thorac Surg 2013;96:857-64.

10. Kormos RL, Teuteberg JJ, Pagani FD, et al. Right ventricular failure in patients with the Heartmate II continuous-flow assist device: incidence, risk factors, and effects on outcomes. J Thorac Cardiovas Surg 2010;139:1316-24.

11. Fukamachi K, McCarthy PM, Smedira NG et al. Preoperative risk factors for right ventricular failure after implantable left ventricular assist device insertion. Ann Thorac Surg 1999;68:2181-4.

12. Nakatani T, Sase K, Oshiyama H, et al. Japanese Registry for Mechanically Assisted Circulatory Support; first report. J Heart Lung Transplant 2017;36:1087-96.

13. Mehra M, Naka Y, Uriel N, et al. A fully magnetically levitated circulatory pump for advanced heart failure. $\mathrm{N}$ Engl J Med 2017;376:440-50.

14. Wang Y, Simon MA, Bonde P, et al. Decision tree for the adjuvant right ventricular support in patients receiving a left ventricular assist device. J Heart Lung Transplant 2012;31:140-9.

15. Fitzpatrick JR, Frederick JR, Hiesinger W, et al. Early planned institution of biventricular mechanical circulatory support results in improved outcomes compared with delayed conversion of a left ventricular assist device to a biventricular assist device. J Thorac Cardiovasc Surg 2009;137:971-7.

16. Morgan JA, John R, Lee BJ, et al. Is severe right ventricular failure in left ventricular assist device recipients a risk factor for unsuccessful bridging to transplant and post-transplant mortality. Ann Thorac Surg 2004;77:859-63.

17. Fitzpatrick JR, Frederick JR, Hsu VM, et al. Risk score derived from preoperative data analysis predicts the need for biventricular mechanical circulatory support. J Heart Lung Transplant 2008;27:1286-92.

18. Matthews JC, Koelling TM, Pafgani FD, Aaronson KD. The right ventricular failure risk score a preoperative tool for assessing the risk of right ventricular failure in left ventricular assist device candidates. J Am Coll Cardiol 2008;51:2163-72.

19. Drakos SG, Janicki L, Horne BD, et al. Risk factors predictive of right ventricular failure after left ventricular assist device implantation. Am J Cardiol 2010;105:1030-5. 
20. Copeland JG, Copeland H, Gustafson M, et al. Experience with more than 100 total artificial heart implants. J Thorac Cardiovasc Surg 2012;143:727-34.

21. Copeland JG, Smith RG, Arabia FA, et al. Cardiac replacement with a total artificial heart as a bridge to transplantation. N Engl J Med 2004;351:859-67.

22. Arabia FA, Cantor RS, Koehl DA, et al. Interagency registry for mechanically assisted circulatory support report on the total artificial heart. J Heart Lung Transplant 2018;37:1304-12.

23. Latremouille C, Carpentier A, Leprince P, et al. A

Cite this article as: Chung JS, Emerson D, Megna D, Arabia FA. Total artificial heart: surgical technique in the patient with normal cardiac anatomy. Ann Cardiothorac Surg 2020;9(2):8188. doi: 10.21037/acs.2020.02.09 bioprosthetic total artificial heart for end-stage heart failure: Results from a pilot study. J Heart Lung Transplant 2018;37:33-7.

24. Kleinheyer M, Timms D, Tansley GD, et al. Rapid Speed Modulation of a Rotary Total Artificial Heart Impeller. Artif Organs 2016;40:824-33.

25. Fumoto H, Horvath DJ, Rao S, et al. In vivo acute performance of the Cleveland Clinic self-regulating, continuous-flow total artificial heart. J Heart Lung Transplant 2010;29:21-6. 